\title{
Systemic Therapy for Patients with Resectable Hepatic Colorectal Metastases: Improving Patient Selection
}

\author{
William R. Jarnagin, MD and Michael D'Angelica, MD \\ Department of Surgery, Memorial Sloan-Kettering Cancer Center, New York, NY
}

The past three decades have seen great changes in the management of hepatic colorectal cancer metastases. While resection remains firmly established as the most effective therapy for selected patients, the emergence of more effective chemotherapeutic agents has been a practice changing development and has considerably altered the therapeutic approach to patients with this disease.

In the palliative setting, the power of contemporary systemic agents is clear, with superior tumor response rates and prolonged survival well documented. Indeed, this greater efficacy translates into conversion to resectability in a significant proportion of patients with advanced, initially unresectable disease, a feature of systemic therapy only rarely seen in the past. ${ }^{1}$

There is thus little doubt that contemporary systemic chemotherapeutic agents, with their greater antitumor activity and ability to bring more patients to resection, have greatly improved long-term outcome in patients with advanced disease. As a result, there is a perception that advances in chemotherapy offer a similar benefit when used in the adjuvant or perioperative setting. Supporting this is a large body of retrospective data that suggests significantly better posthepatectomy survival over time. In the era before contemporary systemic agents, 5-year survival rates of 25$40 \%$ were reported routinely following hepatic resection. By contrast, several studies have demonstrated significantly greater 5-year survival rates for patients treated more contemporaneously. ${ }^{2-5}$ Of note, these analyses typically show improvements in overall survival over time, whereas changes in disease-free survival rates have been much less impressive.

(C) Society of Surgical Oncology 2013

First Received: 18 September 2013; Published Online: 12 October 2013

W. R. Jarnagin, MD

e-mail: jarnagiw@mskcc.org
On the surface, these data would implicate a major contribution from systemic therapy in leading to better outcomes in patients in patients with resectable disease; however, prospective data do not reflect the same level of benefit seen in patients with advanced disease. In fact, the results of multiple prospective randomized trials examining adjuvant or perioperative chemotherapy after potentially curative hepatic resection have shown no improvement in overall survival. In two trials of adjuvant 5-FU after hepatic resection, a combined analysis of 302 randomized patients was unable to demonstrate significant improvements in recurrence-free or overall survival. ${ }^{6}$ Furthermore, a study of adjuvant FOLFIRI compared with 5-FU after hepatic resection in 306 patients showed absolutely no difference in recurrence free or overall survival. ${ }^{7}$ The most notable adjuvant trial, the EORTC study led by Nordlinger, randomized 364 patients to perioperative FOLFOX or observation following complete resection of four or fewer liver metastases, and showed a marginal benefit in progression free survival at 3 years $(7 \%, p=0.058)$; this improved to $9 \%(p=0.025)$ when the analysis was restricted to resected patients. ${ }^{8}$ An updated analysis showed no improvement in overall survival. ${ }^{9}$

These seemingly discordant results between outcomes in the adjuvant and palliative settings are disappointing and somewhat surprising, particularly those reported in the EORTC study, which compared current, standard agents to a "no chemotherapy" group. Although many oncologists point to these data as justification for the routine use of adjuvant or perioperative systemic chemotherapy after potentially curative resection of colorectal liver metastases, the benefit, if present at all, is likely to be small, and the weight of published evidence does not necessarily support this approach.

The reasons that these studies do not show a clear benefit of systemic therapy are unclear, but failure to account fully for the heterogeneity of patients with hepatic 
colorectal metastases is likely a major contributing factor. Although these patients all have stage IV disease, by definition, variability in the risk of disease recurrence and survival after resection is well known. Underscoring this point very clearly, a number of scoring systems have been published over the years that stratify patients effectively into high- and low-risk groups based solely on clinicopathologic variables related to the primary and metastatic disease. ${ }^{10-15}$ Previously, adjuvant or perioperative systemic therapy has not factored into these systems, primarily because of limited options and efficacy and the inability to reliably analyze the uncontrolled use of pre- and/or postoperative systemic therapy. In this era of more effective systemic agents, however, the time has come to investigate means of targeting systemic therapy to patients who are most likely to benefit. Such studies are necessary and would represent a significant advance.

In this issue of Annals of Surgical Oncology, Hirokawa et al. ${ }^{16}$ report a simplified approach to risk stratification in patients undergoing resection of hepatic colorectal metastases that not only correlates with long-term outcome but also predicts the effectiveness of adjuvant (i.e., posthepatectomy) systemic chemotherapy. In this study, the 5-year survival of patients in the low-risk group was similar in those treated with adjuvant chemotherapy compared with those who received no adjuvant therapy. By contrast, the use of adjuvant therapy was associated with an apparent survival advantage when used in high-risk patients.

The study by Hirokawa et al. ${ }^{16}$ has a number of limitations that preclude definitive conclusions, including small sample size, lack of standardized chemotherapeutic protocols, and its retrospective design. Nevertheless, the concept is compelling and should serve as an impetus for more investigation in order to improve the current approach to adjuvant and perioperative treatment in patients with resectable hepatic colorectal metastases. Ultimately, molecular profiling will provide the insight into disease biology necessary to stratify risk reliably and guide therapeutic decisions. Until such time, however, we must rely on variables derived from robust clinical datasets, and future randomized, controlled trials should strongly consider stratification by some risk scoring system.

\section{REFERENCES}

1. Power DG, Kemeny NE. Chemotherapy for the conversion of unresectable colorectal cancer liver metastases to resection. Crit Rev Oncol Hematol. 2011;79(3):251-64.
2. Chan KM, Chiang JM, Lee CF, et al. Outcomes of resection for colorectal cancer hepatic metastases stratified by evolving eras of treatment. World J Surg Oncol. 2011;9:174.

3. House MG, Ito H, Gönen M, et al. Survival after hepatic resection for metastatic colorectal cancer: trends in outcomes for 1,600 patients during two decades at a single institution. $J$ Am Coll Surg. 2010;210:744-52, 752-5.

4. Choti MA, Sitzmann JV, Tiburi MF, et al. Trends in long-term survival following liver resection for hepatic colorectal metastases. Ann Surg. 2002;235:759-66.

5. Andres A, Majno PE, Morel P, et al. Improved long-term outcome of surgery for advanced colorectal liver metastases: reasons and implications for management on the basis of a severity score. Ann Surg Oncol. 2008;15:134-143.

6. Mitry E, Fields AL, Bleiberg H, et al. Adjuvant chemotherapy after potentially curative resection of metastases from colorectal cancer: a pooled analysis of two randomized trials. J Clin Oncol. 2008;26:4906-11.

7. Ychou M, Hohenberger W, Thezenas S, et al. A randomized phase III study comparing adjuvant 5-fluorouracil/folinic acid with FOLFIRI in patients following complete resection of liver metastases from colorectal cancer. Ann Oncol. 2009;20:1964-70.

8. Nordlinger B, Sorbye H, Glimelius B, et al. Perioperative chemotherapy with FOLFOX4 and surgery versus surgery alone for resectable liver metastases from colorectal cancer (EORTC Intergroup trial 40983): a randomised controlled trial. Lancet. 2008;371:1007-16.

9. Nordlinger B, Sorbye H, Glimelius B, et al. EORTC liver metastases intergroup randomized phase III study 40983: longterm survival results. J Clin Oncol. (Meeting Abstracts) 2012; 30(15_Suppl):3508.

10. Nordlinger B, Guiguet M, Vaillant JC, et al. Surgical resection of colorectal carcinoma metastases to the liver. A prognostic scoring system to improve case selection, based on 1568 patients. Association Française de Chirurgie. Cancer. 1996;77:1254-62.

11. Fong Y, Fortner J, Sun RL, et al. Clinical score for predicting recurrence after hepatic resection for metastatic colorectal cancer: analysis of 1001 consecutive cases. Ann Surg. 1999;230:309-18.

12. Iwatsuki S, Dvorchik I, Madariaga JR, et al. Hepatic resection for metastatic colorectal adenocarcinoma: a proposal of a prognostic scoring system. J Am Coll Surg. 1999;189:291-9.

13. Nagashima I, Takada T, Matsuda K, et al. A new scoring system to classify patients with colorectal liver metastases: proposal of criteria to select candidates for hepatic resection. $J$ Hepatobiliary Pancreat Surg. 2004;11:79-83.

14. Rees M, Tekkis PP, Welsh FK, et al. Evaluation of long-term survival after hepatic resection for metastatic colorectal cancer: a multifactorial model of 929 patients. Ann Surg. 2008;247:12535.

15. Konopke R, Kersting S, Distler M, et al. Prognostic factors and evaluation of a clinical score for predicting survival after resection of colorectal liver metastases. Liver Int. 2009;29:89-102.

16. Hirokawa F, Hayashi M, Miyamoto Y, et al. Reconsideration of the indications for adjuvant chemotherapy for liver metastases from colorectal cancer after initial hepatectomy. Ann Surg Oncol. 2013 (in press). 